\title{
Improvement of Adipose Macrophage Polarization in High Fat Diet-Induced Obese GHSR Knockout Mice
}

\author{
Fang Yuan, ${ }^{1}$ Jian $\mathrm{Ma}^{2}$ Xinxin Xiang $\mathbb{D},{ }^{1,3}$ He Lan, ${ }^{1,4}$ Yanhui Xu $\mathbb{D}^{1}$, \\ Jing Zhao, ${ }^{1}$ Yin $\mathrm{Li}\left(\mathbb{D},{ }^{1}\right.$ and Weizhen Zhang ${ }^{1,5}$ \\ ${ }^{1}$ Department of Physiology and Pathophysiology, Peking University Health Science Center; Key Laboratory of \\ Molecular Cardiovascular Science, Ministry of Education, Beijing 100191, China \\ ${ }^{2}$ Department of Geriatrics, No. 401 Hospital of PLA, Qingdao 266071, China \\ ${ }^{3}$ Department of Pathology, Central Hospital of Zibo, Zibo 255000, China \\ ${ }^{4}$ Department of Clinical Laboratory, Xuanwu Hospital, Capital Medical University, Beijing 100053, China \\ ${ }^{5}$ Department of Surgery, University of Michigan, Ann Arbor, MI, USA
}

Correspondence should be addressed to Yin Li; yinli@bjmu.edu.cn

Received 12 February 2018; Revised 6 May 2018; Accepted 28 May 2018; Published 10 July 2018

Academic Editor: Flavia Prodam

Copyright (C) 2018 Fang Yuan et al. This is an open access article distributed under the Creative Commons Attribution License, which permits unrestricted use, distribution, and reproduction in any medium, provided the original work is properly cited.

\begin{abstract}
Purpose. Adipose tissue inflammation is the key linking obesity to insulin resistance. Over $50 \%$ of the interstitial cells in adipose tissue are macrophages, which produce inflammatory cytokines and therefore play an important role in the progression of insulin resistance. Within this classification view, macrophage biology is driven by two polarization phenotypes, $\mathrm{M}_{1}$ (proinflammatory) and $\mathrm{M}_{2}$ (anti-inflammatory). The unique functional receptor of ghrelin, growth hormone secretagogue receptor (GHSR), is a classic seven-transmembrane $G$ protein-coupled receptor that is linked to multiple intracellular signaling pathways. Knockout of GHSR improves the obesity and glucose metabolic disorders, suggesting a crucial role of ghrelin activity in insulin resistance. Here, we discussed whether macrophage polarization phenotypes in adipose tissue were changed in GHSR knockout (GHSR-/-) mice. Methods. GHSR-/- mice were fed with normal chow diet (NCD) or high fat diet (HFD). Markers of different macrophage polarization phenotypes were detected by real-time RT-PCR. Results. The size of adipocytes decreased and interstitial cells, especially infiltrated macrophages, reduced in epididymal adipose tissue of GHSR-/- mice fed with HFD. Compared with wild type mice, the mRNA levels of inflammatory adipokines such as resistin, IL-6, and PAI-1 were significantly lower in epididymal adipose tissue of GHSR-/- mice, whereas anti-inflammatory adipokine, adiponectin, was significantly higher. $\mathrm{M}_{1}$ markers, $\mathrm{MCP}-1$, TNF- $\alpha$, and iNOS, were significantly lower in epididymal adipose tissue of GHSR-/- mice, whereas $\mathrm{M}_{2}$ markers, Arg-1, Mgl-1, were Mrcl, were significantly higher. Conclusion. The GHSR-/- mice fed with HFD showed suppressed adipose inflammation, reduced macrophage infiltration, and enhanced $\mathrm{M}_{2}$ polarization of macrophages in adipose tissue, which improved insulin sensitivity.
\end{abstract}

\section{Introduction}

As the natural ligand of the orphan growth hormone secretagogue receptor type la (GHSRla) [1,2], ghrelin is a 28-amino acid peptide which is secreted by gastric oxyntic glands and octanoylated by ghrelin O-acyl transferase (GOAT), the only enzyme that has been identified [3-5], which is expressed predominantly in the stomach, gut, and pancreas and also at other sites [6]. Human ghrelin mRNA codes for a 117 amino acid peptide, preproghrelin [7], which undergoes endoproteolytic processing and posttranslational modification to produce acyl ghrelin, des-acyl ghrelin, and obestatin [8-12]. Serum acyl ghrelin levels increase with fasting, suggesting that it is an orexigenic hormone involved in meal initiation $[13,14]$. Acyl ghrelin regulates somatic growth, feeding behavior, and energy homeostasis in mammals $[15,16]$, and it also stimulates motility of the stomach and small intestine and acid secretion in rats $[4,17]$. In our previous study, we found that GHSR knockout improved glucose metabolism in skeletal muscle in mice [18].

Insulin resistance is the common basis in pathogenesis for obesity-related diseases [19], such as metabolic syndrome, 
type 2 diabetes, and atherosclerosis, which are low-grade aspecific proinflammatory states. Reciprocal causation of insulin resistance, obesity, and inflammation indicates that inflammatory factors, chemokines, and immunocytes could reduce insulin sensitivity directly or indirectly, participating in the occurrence and development of insulin resistance.

As immune cells, macrophages are pluripotent and play an important role in innate immunity. Macrophage polarization is considered as phenotypic heterogeneity of the mononuclear phagocyte system [20]. As a result of cellular differentiation, widespread tissue distribution, and responsiveness to many endogenous and exogenous stimuli, macrophages are divided into classical or $\mathrm{M}_{1}$ and alternative or $M_{2}$ polarization. $M_{1}$ macrophages are also named proinflammatory macrophages, and $\mathrm{M}_{2}$ macrophages are named anti-inflammatory macrophages; the former secret proinflammatory factors and have proinflammatory functions, and the later have anti-inflammatory functions and are involved in tissue repair [21].

During obesity, the macrophage infiltration in adipose tissue is enhanced, and mainly infiltrated phenotype is $M_{1}$ [21]. The interaction between $M_{1}$ macrophages and adipocytes promotes the secretion of inflammatory factors, which results in the insulin resistance. Suppression of inflammation or inducing transformation of macrophages in adipose tissue from $M_{1}$ to $M_{2}$ can relieve insulin resistance and improve related metabolic syndrome.

In the present study, we investigated the macrophage polarization in the adipose tissue of GHSR knockout mice and contributed to a new mechanism of GHSR knockoutimproved insulin sensitivity.

\section{Materials and Methods}

2.1. Materials. Acyl ghrelin was purchased from Phoenix Pharmaceuticals Inc. (Burlingame, CA). Rabbit antiphospho-AKT (Ser473) and AKT antibodies were from Cell Signaling Technology (Beverly, MA). Rat anti-LAMP-2 (Mac3/84) antibody and chicken anti-rabbit fluorescein isothiocyanate-conjugated IgG were from Santa Cruz Biotechnology Inc. (Santa Cruz, CA). DyLigh 594-goat anti-rat IgG was from EarthOx LLC (EarthOx, CA).

2.2. Ethical Approval. The animals used in this study were handled in accordance with the Guide for the Care and Use of Laboratory Animals published by the US National Institutes of Health (NIH publication no. 85-23, revised 1996), and all the experimental protocols were approved by the Animal Care and Use Committee of Peking University.

2.3. Animals and Treatments. C57BL/6J mice, Ghsrla knockout (GHSR-/-) mice, were used in the present study. Ghsrla gene knockout mice in which exon 1 and exon 2 had been deleted were obtained from the Shanghai Research Center for Biomodel Organisms (Shanghai, China) [18, 22]. Deletion of the Ghsrla gene fragment was confirmed by the absence of relative gene products examined by RT-PCR. 6week-old male mice were housed in specific pathogen-free microisolators and maintained in a regulated environment $\left(24^{\circ} \mathrm{C}, 12 \mathrm{~h}\right.$ light-dark cycle, with lights on at 07:00 AM). Regular chow and water were available ad libitum. Mice were assigned to receive standard laboratory chow (NCD) or a high fat diet (HFD) (45\% fat, D12451; Research Diets, New Brunswick, NJ, USA) for 12 weeks.

2.4. Cell Culture. RAW264.7 cells, the murine peritoneal macrophage-like cell line, were cultured in growth medium (high-glucose Dulbecco's modified Eagle's medium (DMEM); Invitrogen, Grand Island, NY, USA) supplemented with $10 \%$ heat-inactivated fetal bovine serum (FBS; US Biotechnologies), 100 units/ml penicillin, and 100 units/ml streptomycin (Invitrogen) and incubated at $37^{\circ} \mathrm{C}$ with $5 \% \mathrm{CO}_{2}$. Cells were passaged weekly after trypsin-EDTA detachment. All studies were performed on RAW264.7 cells at passages 20-25. Then cells were cultured with LPS $(10 \mathrm{ng} / \mathrm{ml})$ or IL-4 $(10 \mathrm{ng} / \mathrm{ml})$ to generate classically $\left(M_{1}\right)$ or alternatively $\left(M_{2}\right)$ polarized cells.

2.5. Glucose Tolerance Test. Glucose tolerance test was performed as described [18]. Blood was drawn from a cut at the tip of the tail at $0,30,60,90$, and $120 \mathrm{~min}$, and glucose concentrations were detected immediately with Glucotrend from Roche Diagnostics (Mannheim, Germany) according to the manufacturer's instructions.

2.6. RNA Extraction and Quantitative Real-Time PCR Analysis. Total RNA was isolated using the Trizol reagent. Reverse transcription and quantitative real-time PCR were performed as previously described [23-25]. PCR was conducted in a $25 \mu \mathrm{l}$ volume containing $2.5 \mathrm{ng} \mathrm{cDNA}, 5 \mathrm{mM} \mathrm{MgCl}_{2}, 0.2 \mathrm{mM}$ dNTPs, $0.2 \mu \mathrm{M}$ each primer, $1.25 \mathrm{U}$ AmpliTaq Polymerase, and $1 \mu \mathrm{l} 800 \mathrm{x}$ diluted SYBRGreen I stock using the Mx3000 multiplex quantitative PCR system (Stratagene, La Jolla, CA). The PCR program was as follows: holding $95^{\circ} \mathrm{C}$ for $7 \mathrm{~min} ; 95^{\circ} \mathrm{C}$ for $30 \mathrm{~s}, 60^{\circ} \mathrm{C}$ for $35 \mathrm{~s}$, and $72^{\circ} \mathrm{C}$ for $35 \mathrm{~s}$. mRNA expression was quantified using the comparative cross threshold (CT) method. The CT value of the housekeeping gene $\beta$-actin was subtracted from the CT value of the target gene to obtain ${ }^{\triangle} \mathrm{CT}$. The normalized fold changes of detected gene' mRNA expression were expressed as $2{ }^{-}{ }^{\Delta \Delta C T}$, where $\Delta \Delta \mathrm{CT}=\Delta \mathrm{CT}$ sample $-\Delta \mathrm{CT}$ control. PCR reactions were performed in duplicate and each experiment was repeated for 3-5 times. Primers used in this study were shown in Table 1.

2.7. Western Blot Analysis. As previously described [23-25], the adipose tissue and cultured cells were quickly harvested, rinsed thoroughly with PBS, and then homogenized on ice in lysis buffer (50 mM Tris-Cl, 15 mM EGTA, $100 \mathrm{mM} \mathrm{NaCl}$, $0.1 \%$ Triton X-100 supplemented with protease inhibitor cocktail, $\mathrm{pH}$ 7.5). After centrifugation for $10 \mathrm{~min}$ at $4^{\circ} \mathrm{C}$, the supernatant was used for western blot analysis. Protein concentration was measured by Bradford's method. A total of $60 \mu \mathrm{g}$ protein from each sample was loaded onto SDS-PAGE gel. Proteins were transferred to polyvinylidene fluoride membranes. The membranes were incubated for $1 \mathrm{~h}$ at room temperature with $5 \%$ fat-free milk in Tris buffered saline containing Tween-20, followed by incubation overnight at $4^{\circ} \mathrm{C}$ with the individual primary antibody. Specific reaction was detected using IRDye-conjugated second antibody and visualized using the Odyssey infrared imaging system 
TABLE 1: List and sequence of primers.

\begin{tabular}{lcc}
\hline & Upstream primer $\left(5^{\prime}-3^{\prime}\right)$ & Downstream primer $\left(5^{\prime}-3^{\prime}\right)$ \\
\hline Resistin & TCCTTGTCCCTGAACTGC & ACGAATGTCCCACGAGC \\
Adiponectin & ACCAGTATCAGGAAAAGAATGT & TAGAGAAGAAAGCCAGTAAATG \\
IL-6 & AGTTGTGCAATGGCAATTCTG & GGAAATTGGGGTAGGAAGGAC \\
PAI-1 & CCTCACCAACATCTTGGATGCT & TGCAGTGCCTGTGCTACAGAGA \\
MCP-1 & ACTGAAGCCAGCTCTCTCTTCCTC & TTCCTTCTTGGGTCAGCACAGAC \\
TNF- $\alpha$ & CGTCGTAGCAAACCACCAAG & GAGATAGCAAATCGGCTGACG \\
iNOS & CCAAGCCCTCACCTACTTCC & CTCTGAGGGCTGACACAAGG \\
Arg-1 & CTCCAAGCCAAAGTCCTTAGAG \\
Mgl-1 & TGAGAAAGGCTTTAAGAACTGGG & AGGAGCTGTCATTAGGGACATC \\
Mrc-1 & AAACACAGACTGACCCTTCCC & GACCACCTGTAGTGATGTGGG \\
$\beta$-actin & ATCTGGCACCACACCTTC & GTTAGTGTACCGCACCCTCC \\
\hline
\end{tabular}

(LI-COR Biosciences, Lincoln, NE). Quantification of image density in pixel was performed by using the Odyssey infrared imaging system (LI-COR Biosciences, Lincoln, NE).

2.8. HぬE Histology. Mice were deeply anesthetized using pentobarbital. The epididymal adipose tissue was quickly removed and rinsed thoroughly with PBS. The tissue was fixed in $4 \%$ paraformaldehyde, dehydrated, embedded in wax, and sectioned at $6 \mu \mathrm{m}$. Diameter of each adipocyte in each field was measured using image analysis software Image J (v1.48). For each group, cell sizes of about 450 adipocytes from 3-4 mice were measured and plotted as histograms.

2.9. Immunofluorescence. Mice were deeply anesthetized using pentobarbital. The same part of adipose tissue was quickly removed and rinsed thoroughly with PBS. The tissue was fixed in $4 \%$ paraformaldehyde, dehydrated, embedded in wax, and sectioned at $6 \mu \mathrm{m}$. Paraffin-embedded sections were dewaxed, rehydrated, and rinsed in PBS. After boiling for $10 \mathrm{~min}$ in $10 \mathrm{mM}$ sodium citrate buffer ( $\mathrm{pH} 6.0$ ), sections were blocked in 1\% BSA in PBS for $1 \mathrm{~h}$ at room temperature, then incubated overnight at $4^{\circ} \mathrm{C}$ with LAMP-2 (Mac3/84) antibody alone, and then washed in $1 \mathrm{X}$ PBS/0.1\% Tween-20 three times for 5 minutes each. Tissue sections were then incubated at room temperature for $1 \mathrm{~h}$ with the following secondary antibodies (DyLigh 594-goat anti-rat IgG). Controls included substituting primary antibody with rat IgG. The nuclei were visualized by staining with Hoechst 33258 for $10 \mathrm{~min}$. Photomicrographs were taken under a confocal laserscanning microscope (Leica, Germany).

2.10. Statistical Analysis. Data were expressed as means \pm SEM. Data analysis used GraphPad Prism software. Oneway ANOVA, Student-Newman-Keul's test (comparisons between multiple groups), or unpaired Student's $t$-test (between two groups) was used as appropriate. P value $<0.05$ denotes statistical significance.

\section{Results}

3.1. Improvement of Glucose Metabolism in GHSR-/- Mice. GHSR-/- mice and wild type (WT) mice were fed with normal chow diet (NCD) or high fat diet (HFD) separately. The food and water intake of mice were shown in Supplemental Figure 1. Food intake was less in mice fed the HF diet, especially in GHSR-/- mice. The body weight of wild type mice fed with HFD increased gradually in 4 weeks, while the GHSR-/mice showed no significant differences in the body weight with different diet (Figure 1(a)). We also found that GHSR-/mice showed less epididymal fat weight (Figure 1(b)) and fat/body weight ratio (Figure 1(c)) than WT mice when fed with HFD.

We further examined insulin sensitivity by performing glucose tolerance test. Wild type mice fed with high fat diet demonstrated severe hyperglycemia upon administration of glucose and impaired glucose tolerance, with typical glucose disposal curves of insulin resistance, whereas the glucose metabolism was not affected by high fat diet in GHSR-/- mice (Figure 1(d)). Insulin-induced Akt phosphorylation (Ser473) was significantly inhibited in wild type mice fed with HFD, but not in the GHSR-/- mice (Figure 1(e)). Therefore, deletion of GHSR could improve the glucose metabolic disorders induced by high fat diet.

3.2. Improvement of Adipose Tissue Inflammation in GHSR-/Mice. It has been reported that insulin resistance and inflammation are intertwined and interdependent, whereas obesity is considered as a low-grade aspecific proinflammatory state. The above results indicated that obesity and glucose metabolic disorders induced by high fat diet were improved in GHSR-/- mice. We then detected the expression of inflammatory adipokines, such as resistin, interleukin6 (IL-6), and Plasminogen activator inhibitor-1 (PAI-1), in epididymal adipose tissue. Compared with wild type mice fed with high fat diet, the mRNA levels of resistin and IL-6 were significantly lower in epididymal adipose tissues of GHSR-/- mice fed with high fat diet, whereas anti-inflammatory adipokine, adiponectin, was significantly higher (Figure 2(a)). H\&E staining showed that the size of adipocytes decreased significantly and crown-like structure also reduced in epididymal adipose tissues of GHSR-/- mice fed with high fat diet (Figure 2(b)).

More than $50 \%$ of the interstitial cells in adipose tissue are macrophages, which are sensitive to the chemokines produced by adipose tissue under inflammatory state and can 


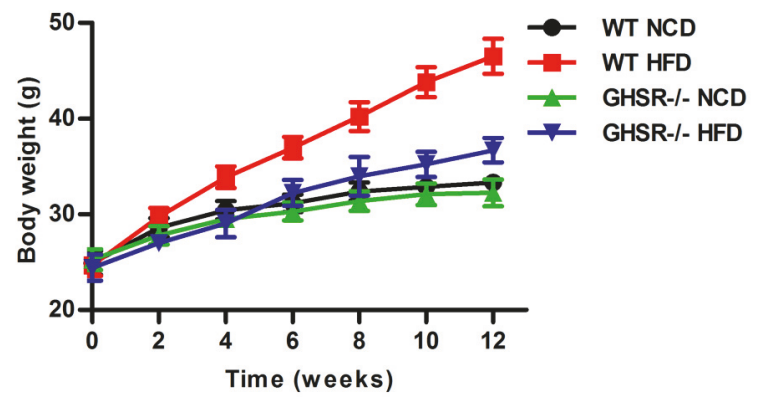

(a)

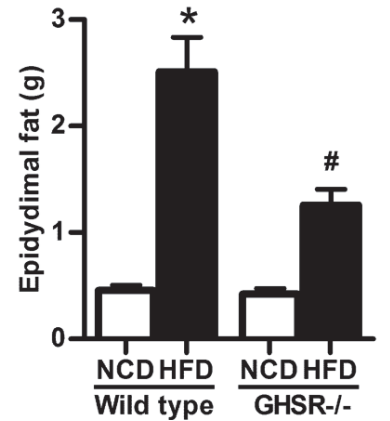

(b)

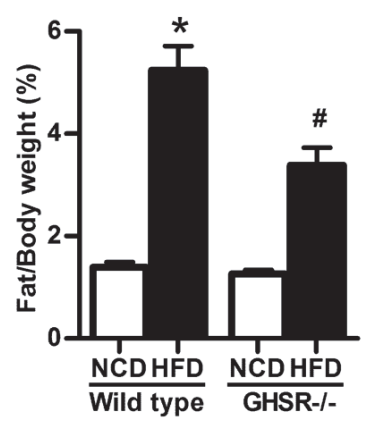

(c)

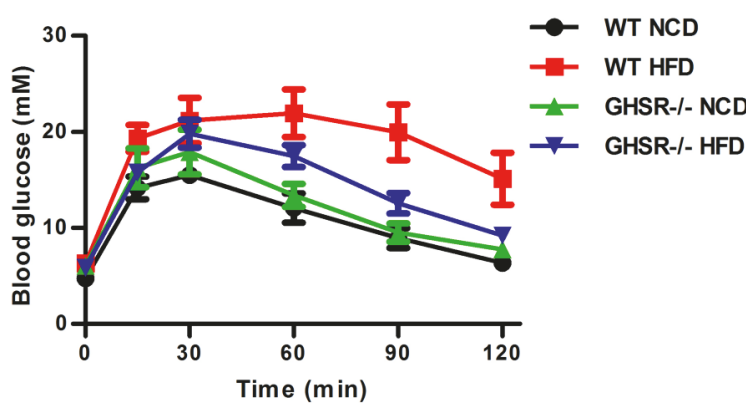

(d)

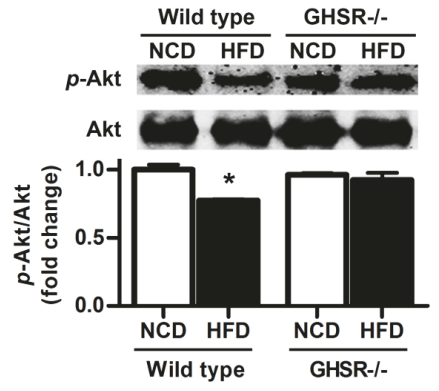

(e)

FIGURE 1: Improvement of glucose metabolism in GHSR knockout mice. (a) Body weight of GHSR knockout (GHSR-/-) mice and wild type (WT) mice fed with different diet. (b) The average daily food intake of GHSR-/- mice and wild type (WT) mice fed with normal chow diet or high fat diet for 12 weeks. (c) The average daily water intake of GHSR-/- mice and wild type mice fed with normal chow diet or high fat diet. (d) Results of glucose tolerance test using GHSR knockout mice and wild type mice fed with normal chow diet (NCD) or high fat diet (HFD) for 12 weeks. All results were expressed as means \pm SEM. (e) Western blotting results from GHSR knockout mice and wild type mice fed with normal chow diet or high fat diet for 12 weeks. Insulin at a dose of $2 \mathrm{IU} / \mathrm{kg}$ was injected intraperitoneally 15 min before harvest of the adipose tissue; phospho-Akt (Ser473) and Akt in adipose tissue were detected using specific antibodies. Total Akt level was used as internal control. Quantification of image analysis of $p$-Akt/Akt was expressed as means \pm SEM. $*$ denotes $\mathrm{P}<0.05$ compared with wild type mice fed with NCD; ${ }^{\#}$ denotes $\mathrm{P}<0.05$ compared with WT mice fed with HFD.

infiltrate in adipose tissue through chemotactic migration. Therefore, we then observed the variation of macrophages in epididymal adipose tissues of wild type mice and GHSR-/mice by immunofluorescence. We found that Mac-3 staining, the marker of macrophages, significantly reduced in epididymal adipose tissues of GHSR-/- mice fed with high fat diet (Figure 2(c)), indicating the ameliorated macrophage infiltration and adipose inflammation.

\subsection{Macrophage Polarization in Epididymal Adipose Tissue} of GHSR-/- Mice. Macrophage activation has been operationally defined as two antipodal polarization states, $\mathrm{M}_{1}$ (proinflammatory) and $\mathrm{M}_{2}$ (anti-inflammatory), which have different functions. We chose corresponding markers to detect the changes of macrophage polarization in epididymal adipose tissues of wild type mice and GHSR-/- mice with normal chow diet and high fat diet. We observed that, compared with normal chow diet, high fat diet significantly induced the mRNA levels of $M_{1}$ markers, such as monocyte chemotactic protein-1 (MCP-1), tumor necrosis factor $\alpha$ (TNF- $\alpha$ ), and inducible nitric oxide synthase (iNOS) in wild type mice (Figure 3(a)), while the $\mathrm{M}_{2}$ markers, like arginase1 (Arg-1) and macrophage galactose-type lectin-1 (Mgl-1)
(Figure 3(b)), decreased markedly, but mannose receptor C type $1(\mathrm{Mrcl})$ remained unchanged. However, these trends were turned over in GHSR-/- mice (Figures 3(a) and 3(b)). All the results above indicated that deletion of GHSR could inhibit the adipose tissue inflammation, decrease the macrophage infiltration, and promote the macrophages polarization to $\mathrm{M}_{2}$.

3.4. Effect of Acyl Ghrelin on RAW264.7 Cells Polarization In Vitro. As reported, acyl ghrelin does its functions by binding with its receptor, GHSRla. Therefore, the improvement of adipose tissue inflammation, decreased macrophage infiltration, and macrophages polarization in GHSR-/- mice might be due to the effect of acyl ghrelin. We then detected the direct effect of acyl ghrelin on the polarization of RAW264.7 murine macrophage cells, under the stimulation of LPS or IL-4, the classical polarizing inducer of $\mathrm{M}_{1}$ or $\mathrm{M}_{2}$. Arg-1 was chosen as $\mathrm{M}_{2}$ marker and MCP-1 was chosen as $\mathrm{M}_{1}$ marker. We found that acyl ghrelin could enhance the effect of LPS on increasing MCP-1 and decreasing Arg-1 mRNA levels (Figure 4(a)), while weakening the effect of IL-4 on increasing Arg-1 and decreasing MCP-1 mRNA levels (Figure 4(b)), which indicated the proinflammatory effect of acyl ghrelin. 


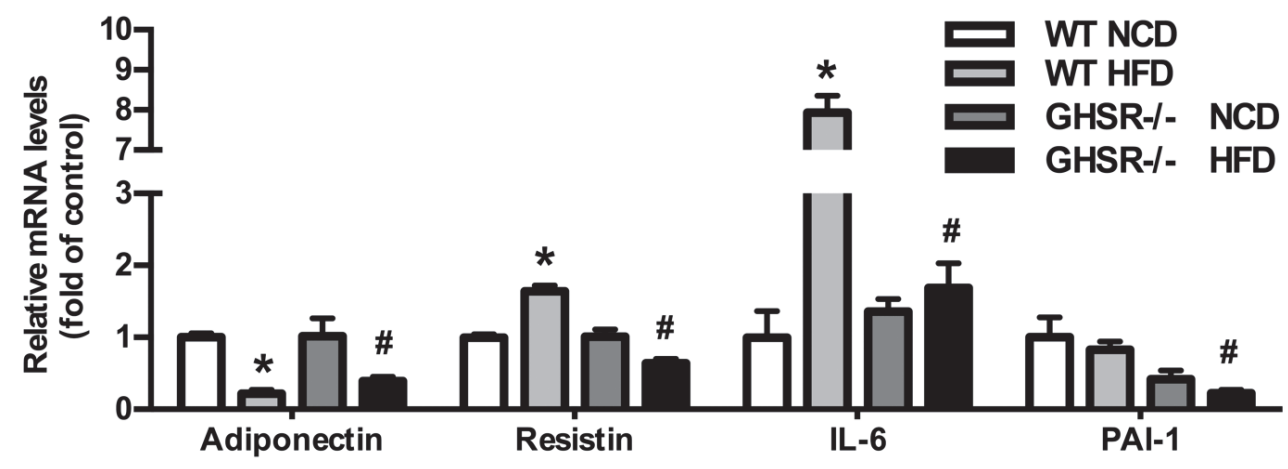

(a)
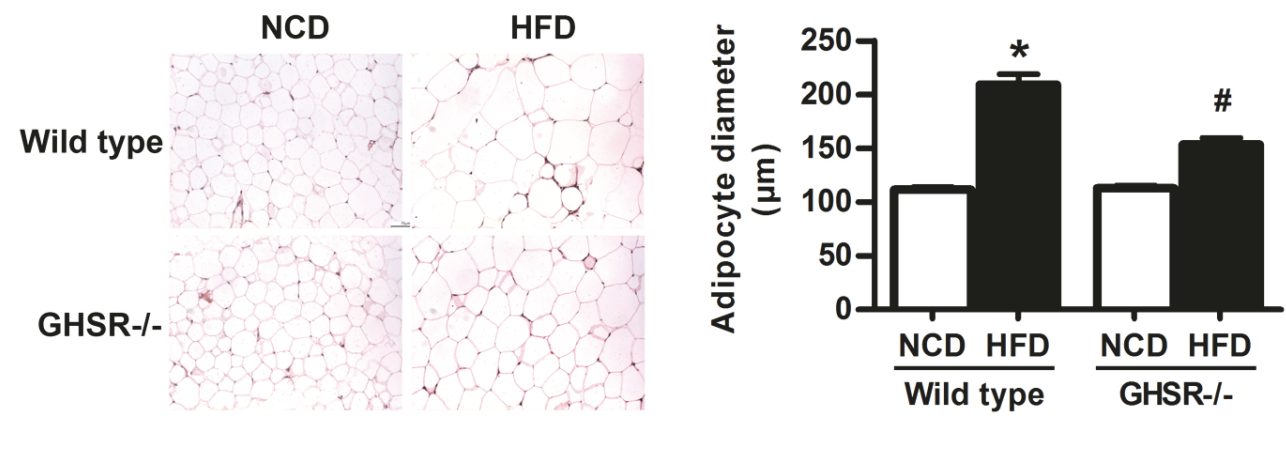

(b)
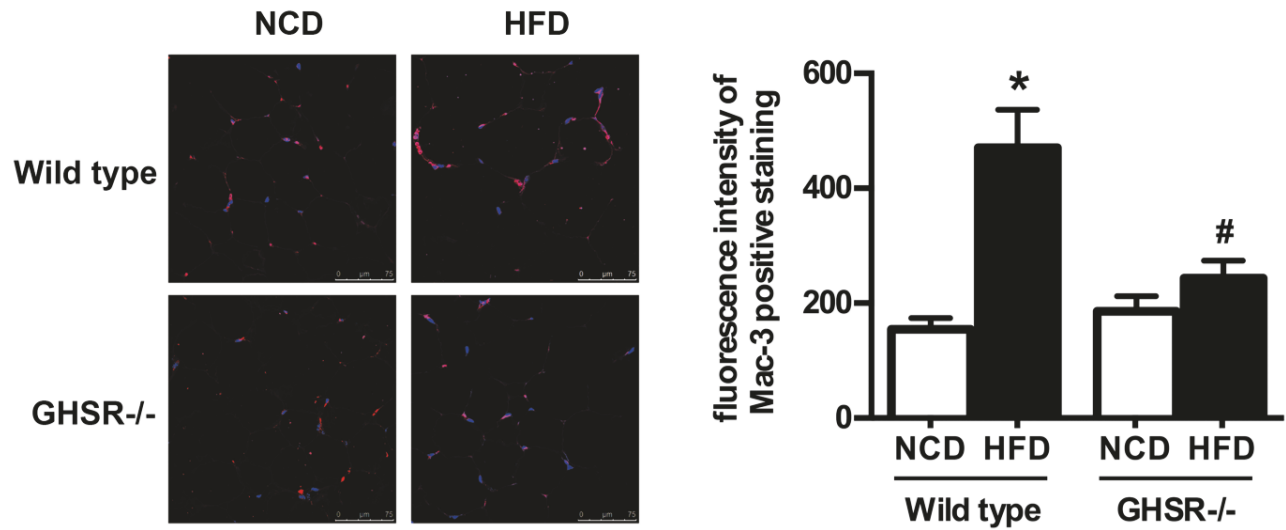

(c)

FIGURE 2: Improvement of adipose tissue inflammation in GHSR knockout mice. (a) Expression of inflammatory adipokines in epididymal adipose tissue of GHSR knockout mice, such as anti-inflammatory adipokine adiponectin and proinflammatory adipokines resistin, IL-6, and PAI-1. Relative mRNA levels were normalized to the levels for wild type mice fed with normal chow diet. Data are means \pm SEM, $n=5$. (b) Adipocytes volume and interstitial cell infiltration detected by H\&E staining. (c) Macrophage infiltration detected by immunofluorescence histochemistry staining of Mac-3. * denotes $\mathrm{P}<0.05$ compared with WT mice fed with NCD; ${ }^{*}$ denotes $\mathrm{P}<0.05$ compared with WT mice fed with HFD.

These results suggest that acyl ghrelin could improve macrophage polarization under inflammatory state. Combined with the results in vivo, it can be concluded that deletion of GHSR would abolish the effect of acyl ghrelin, then inhibit the adipose tissue inflammation, decrease the macrophage infiltration, promote the macrophages polarization to $\mathrm{M}_{2}$, and finally improve insulin sensitivity and glucose metabolism.

\section{Discussion}

In the present study, we reported that GHSR knockout mice fed with HFD showed improved insulin sensitivity through improved adipose inflammation. This conclusion is supported by the following observations: (1) GHSR-/- mice fed with HFD showed suppressed expression of inflammatory adipokine in adipose tissue; (2) GHSR-/- mice fed with 


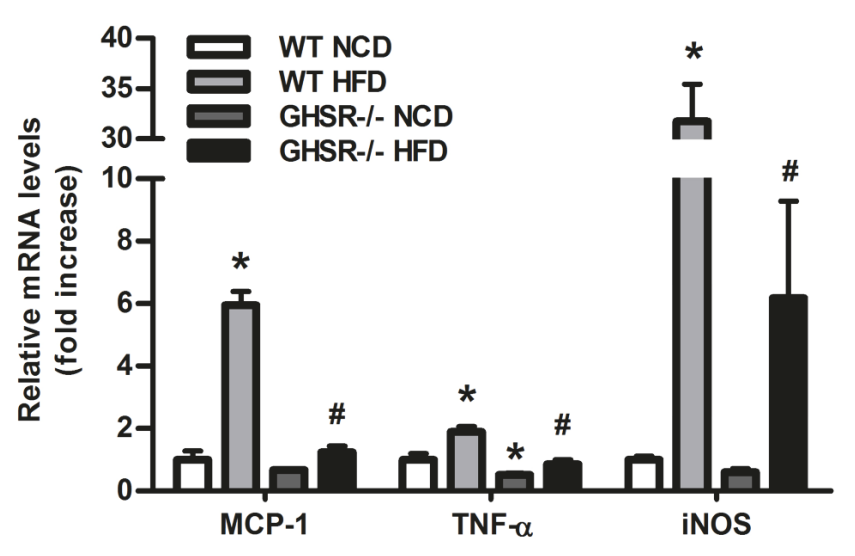

(a)

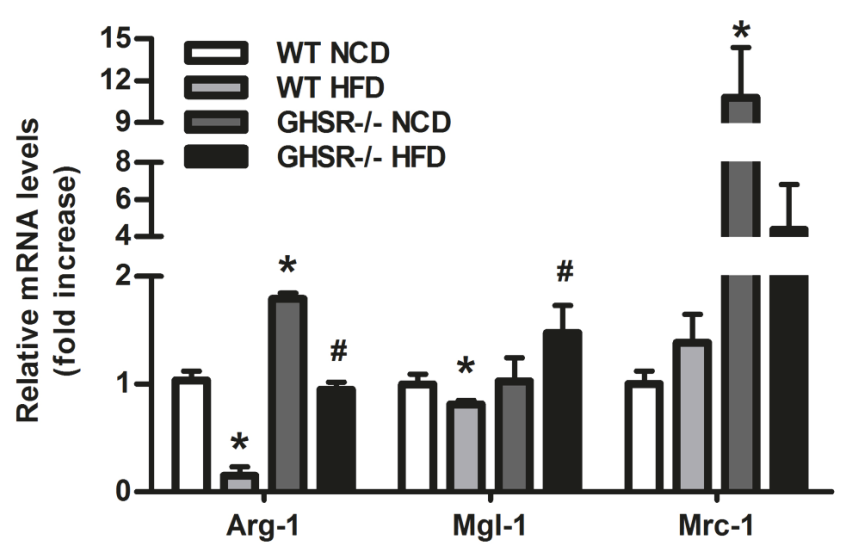

(b)

FIGURE 3: Macrophage polarization in epididymal adipose tissue of GHSR knockout mice. The mRNAs were extracted from the epididymal adipose tissue harvested from the GHSR knockout mice and wild type mice fed with different diet. Real-time PCR was performed to evaluate the expression of macrophage specific markers. (a) $\mathrm{M}_{1}$ markers MCP-1, TNF- $\alpha$, iNOS and (b) $\mathrm{M}_{2}$ markers Arg-1, Mgl-1, Mrcl were detected. Data are means \pm SEM $n=5$. * denotes $\mathrm{P}<0.05$ compared with WT mice fed with $\mathrm{NCD} ;{ }^{*}$ denotes $\mathrm{P}<0.05$ compared with WT mice fed with HFD.

HFD showed reduced macrophage infiltration in adipose tissue; (3) GHSR-/- mice fed with HFD showed M2 polarization of macrophages in adipose tissue; (4) acyl ghrelin enhanced $\mathrm{M}_{1}$ polarization of RAW 264.7 macrophages under inflammatory status. To the best of our knowledge, this is the first report demonstrating the involvement of adiposeinfiltrated macrophage polarization in the improvement of insulin sensitivity of GHSR knockout mice fed with HFD.

Ghrelin regulates processes associated with cancer, including cell proliferation, apoptosis, cell migration, cell invasion, inflammation, and angiogenesis [26]. Ghrelin also plays an important role in metabolism. It was reported that ghrelin could inhibit glucose-stimulated insulin secretion in a dose-dependent manner in vitro [27]; intravenous ghrelin injection decreased plasma insulin and increased plasma glucose levels, likely by inhibition of insulin secretion [27]; ablation of ghrelin, GHSR, or GOAT enhanced insulin release and attenuated impaired glucose tolerance in high fat dietinduced and leptin-deficient obese mice models [28]. Clinical studies also showed that infusing ghrelin to a supraphysiologic level would inhibit the glucose-stimulated insulin secretion and insulin sensitivity and would reduce glucose tolerance in humans [29]. In our studies, we also found that ablation of the action of acyl ghrelin by deletion of GHSR showed similar changes such as impaired insulin sensitivity, especially in high fat diet status.

Existing researches showed that, in the early stage of inflammatory diseases, the gradients of some inflammatory mediators play important roles in the recruitment of leukocytes to inflamed areas [30]. Different from the recruited neutrophils with short life spans due to apoptosis [31], macrophages, as immune cells, have longer life spans and play a more important role to clear neutrophils via phagocytosis. Opinions about the effect of ghrelin on inflammation are different; although some studies demonstrated it as a proinflammatory agent [32], most reports indicated that ghrelin acts as an anti-inflammatory factor [33, 34]. The reason might be the different cell lines and research objectives. In our study, we found that acyl ghrelin could promote the macrophages polarization to $\mathrm{M}_{1}$ directly in vitro, and knockout of its receptor GHSRla inhibited adipose tissue inflammation, decreased the macrophage infiltration, and promoted the macrophages polarization to $\mathrm{M}_{2}$. Therefore, acyl ghrelin might exert proinflammatory effect, especially in our high fat diet system, which is consistent with the reports that ghrelin signaling has an important role in macrophage polarization and adipose tissue inflammation during aging [32] or feeding with high fructose corn syrup [35], in which the concurrences of obesity and insulin resistance are significantly greater. Since we have reported that the cytokine intermedin treatment inhibits chronic inflammation and improves systemic insulin sensitivity through restoring the M1/M2 balance in adipose tissues [36], the involvement of adipose-infiltrated macrophage polarization might be the key point in the improvement of insulin sensitivity of GHSR knockout mice fed with HFD.

Besides the direct effect of ghrelin elimination on macrophages polarization, the decrease of food intake in GHSR knockout mice should also be considered, which will in turn promote the resistance to high fat diet-induced obesity $[37,38]$ and adipose inflammation. However, in aging mice, knockout of GHSR did not affect the body weight and food intake [39], although adipose tissue inflammation and insulin resistance were improved [32]. Whether any other factors participate in the improvement of glucose tolerance and inflammatory status in GHSR knockout mice fed with HFD needs further studies.

In conclusion, our results indicated that acyl ghrelin could promote macrophage polarization to $M_{1}$ under inflammatory state in vitro. While in vivo, deletion of GHSR would abolish the effect of acyl ghrelin and subsequently inhibit the adipose tissue inflammation, decrease the macrophage infiltration, promote the macrophages polarization to $\mathrm{M}_{2}$ phenotype, and finally improve insulin sensitivity. Our study will give a new insight about the function of acyl ghrelin and its receptor GHSR. 


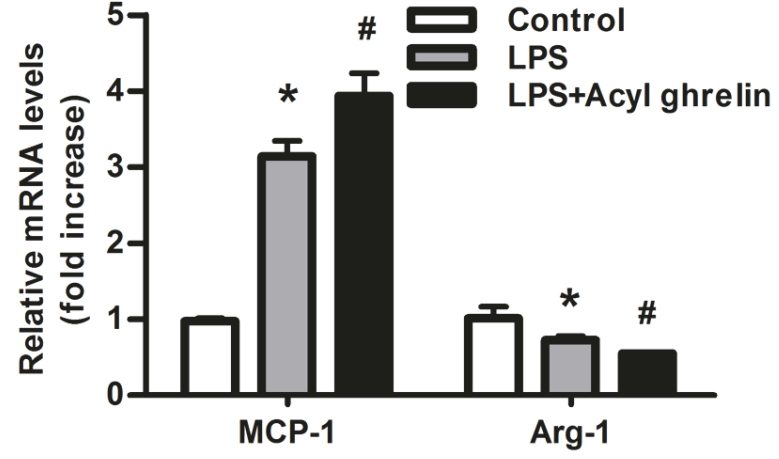

(a)

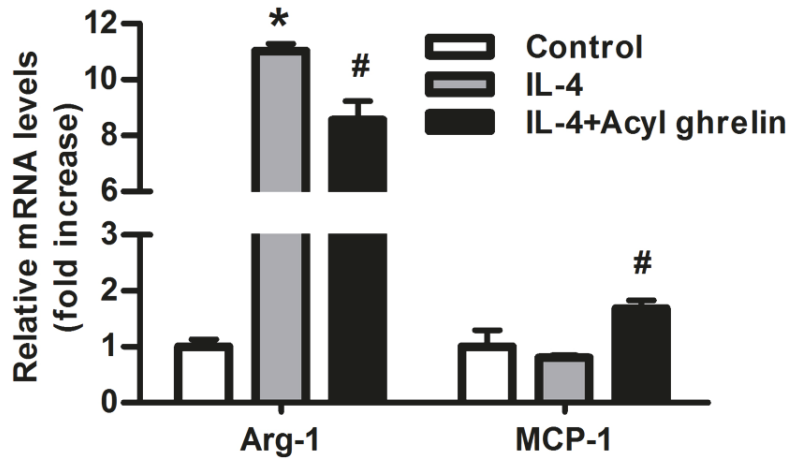

(b)

FIGURE 4: Acyl ghrelin influences the effect of LPS or IL-4 on RAW264.7 cell polarization. The mRNAs were extracted from the RAW 264.7 pretreated with acyl ghrelin for 30 minutes then for 6 hours with LPS (10 ng/ml) (a) or IL-4 (10 ng/ml) (b). Real-time PCR was performed to evaluate the expression of macrophage specific markers Arg- 1 and MCP- 1 . Data are means \pm SEM from 3 separate experiments. $*$ denotes $\mathrm{P}<0.05$ compared with control group; ${ }^{*}$ denotes $\mathrm{P}<0.05$ compared with cells treated with LPS or IL- 4 alone.

\section{Data Availability}

The data used to support the findings of this study are available from the corresponding author upon request.

\section{Disclosure}

A part of the manuscript was presented as poster at the $24^{\text {th }}$ Conference of Chinese Association for Physiological Sciences.

\section{Conflicts of Interest}

The authors declare that they have no conflicts of interest.

\section{Authors' Contributions}

Fang Yuan and Jian Ma contributed equally to this work.

\section{Acknowledgments}

This work was supported by grants from the National Natural Science Foundation of China (81670780 to Yin Li and 81600695 to Xinxin Xiang).

\section{Supplementary Materials}

Online supplementary figure and legend were shown. (Supplementary Materials)

\section{References}

[1] M. Kojima, H. Hosoda, Y. Date, M. Nakazato, H. Matsuo, and $\mathrm{K}$. Kangawa, "Ghrelin is a growth-hormone-releasing acylated peptide from stomach," Nature, vol. 402, no. 6762, pp. 656-660, 1999.

[2] K.-I. Koyama, D. Yasuhara, T. Nakahara et al., "Changes in acyl ghrelin, des-acyl ghrelin, and ratio of acyl ghrelin to total ghrelin with short-term refeeding in female inpatients with restricting-type anorexia nervosa," Hormone and Metabolic Research, vol. 42, no. 8, pp. 595-598, 2010.

[3] J. A. Gutierrez, P. J. Solenberg, D. R. Perkins et al., "Ghrelin octanoylation mediated by an orphan lipid transferase," Proceedings of the National Acadamy of Sciences of the United States of America, vol. 105, no. 17, pp. 6320-6325, 2008.

[4] J. Yang, M. S. Brown, G. Liang, N. V. Grishin, and J. L. Goldstein, "Identification of the Acyltransferase that Octanoylates Ghrelin, an Appetite-Stimulating Peptide Hormone," Cell, vol. 132, no. 3, pp. 387-396, 2008.

[5] P. J. D. Delhanty, Y. Sun, J. A. Visser et al., "Unacylated ghrelin rapidly modulates lipogenic and insulin signaling pathway gene expression in metabolically active tissues of GHSR deleted mice," PLoS ONE, vol. 5, no. 7, Article ID el1749, 2010.

[6] K. Kang, J. Schmahl, J.-M. Lee et al., "Mouse ghrelin-Oacyltransferase (GOAT) plays a critical role in bile acid reabsorption," The FASEB Journal, vol. 26, no. 1, pp. 259-271, 2012.

[7] N. Kanamoto, T. Akamizu, T. Tagami et al., "Genomic structure and characterization of the $5 /$-flanking region of the human ghrelin gene," Endocrinology, vol. 145, no. 9, pp. 4144-4153, 2004.

[8] H. Hosoda, M. Kojima, H. Matsuo, and K. Kangawa, "Purification and characterization of rat des-Gln14-ghrelin, a second endogenous ligand for the growth hormone secretagogue receptor," The Journal of Biological Chemistry, vol. 275, no. 29, pp. 21995-22000, 2000.

[9] H. Hosoda, M. Kojima, T. Mizushima, S. Shimizu, and K. Kangawa, "Structural divergence of human ghrelin: Identification of multiple ghrelin-derived molecules produced by posttranslational processing," The Journal of Biological Chemistry, vol. 278, no. 1, pp. 64-70, 2003.

[10] P. L. Jeffery, R. P. Duncan, A. H. Yeh et al., "Expression of the ghrelin axis in the mouse: An exon 4-deleted mouse proghrelin variant encodes a novel C terminal peptide," Endocrinology, vol. 146, no. 1, pp. 432-440, 2005.

[11] A. H. Yeh, P. L. Jeffery, R. P. Duncan, A. C. Herington, and L. K. Chopin, "Ghrelin and a novel preproghrelin isoform are highly expressed in prostate cancer and ghrelin activates mitogenactivated protein kinase in prostate cancer," Clinical Cancer Research, vol. 11, no. 23, pp. 8295-8303, 2005. 
[12] J. V. Zhang, P.-G. Ren, O. Avsian-Kretchmer et al., "Obestatin, a peptide encoded by the ghrelin gene, opposes ghrelin's effects on food intake," Science, vol. 310, no. 5750, pp. 996-999, 2005.

[13] M. Tschop, D. L. Smiley, and M. L. Heiman, "Ghrelin induces adiposity in rodents," Nature, vol. 407, no. 6806, pp. 908-913, 2000.

[14] D. E. Cummings, J. Q. Purnell, R. S. Frayo, K. Schmidova, B. E. Wisse, and D. S. Weigle, "A preprandial rise in plasma ghrelin levels suggests a role in meal initiation in humans," Diabetes, vol. 50, no. 8, pp. 1714-1719, 2001.

[15] C. Gauna, P. Uitterlinden, P. Kramer et al., "Intravenous glucose administration in fasting rats has differential effects on acylated and unacylated ghrelin in the portal and systemic circulation: A comparison between portal and peripheral concentrations in anesthetized rats," Endocrinology, vol. 148, no. 11, pp. 5278-5287, 2007.

[16] Q. Xu, H. Bian, N. Han, R. Hou, Z. Zhang, and M. Zhu, "CDNA cloning and expression of ghrelin in giant panda (Ailuropoda melanoleuca)," Molecular Biology Reports, vol. 37, no. 6, pp. 2903-2907, 2010.

[17] Y. Date, M. Kojima, H. Hosoda et al., "Ghrelin, a novel growth hormone-releasing acylated peptide, is synthesized in a distinct endocrine cell type in the gastrointestinal tracts of rats and humans," Endocrinology, vol. 141, no. 11, pp. 4255-4261, 2000.

[18] G. Xu, Z. Wang, Y. Li et al., "Ghrelin contributes to derangements of glucose metabolism induced by rapamycin in mice," Diabetologia, vol. 55, no. 6, pp. 1813-1823, 2012.

[19] E. Orgel and S. D. Mittelman, "The links between insulin resistance, diabetes, and cancer," Current Diabetes Reports, vol. 13, no. 2, pp. 213-222, 2013.

[20] N. Leitinger and I. G. Schulman, "Phenotypic polarization of macrophages in atherosclerosis," Arteriosclerosis, Thrombosis, and Vascular Biology, vol. 33, no. 6, pp. 1120-1126, 2013.

[21] C. N. Lumeng, J. L. Bodzin, and A. R. Saltiel, "Obesity induces a phenotypic switch in adipose tissue macrophage polarization," The Journal of Clinical Investigation, vol. 117, no. 1, pp. 175-184, 2007.

[22] W. W. Z. Wang, Y. Zhang, J. Fei, and Q. Zheng, "Generation of mouse embryonic stem cell clones for ghrelin receptor gene knock out," Chinese Journal of Bases and Clinics in General Surgery, pp. 733-736, 2008.

[23] G. Xu, Y. Li, W. An et al., "Gastric mammalian target of rapamycin signaling regulates ghrelin production and food intake," Endocrinology, vol. 150, no. 8, pp. 3637-3644, 2009.

[24] W. An, Y. Li, G. Xu et al., "Modulation of ghrelin oacyltransferase expression in pancreatic islets," Cellular Physiology and Biochemistry, vol. 26, no. 4-5, pp. 707-716, 2010.

[25] Z. Li, G. Xu, Y. Li, J. Zhao, M. W. Mulholland, and W. Zhang, "MTOR-dependent modulation of gastric nesfatin-1/NUCB2," Cellular Physiology and Biochemistry, vol. 29, no. 3-4, pp. 493500, 2012.

[26] L. K. Chopin, I. Seim, C. M. Walpole, and A. C. Herington, "The Ghrelin axis-does it have an appetite for cancer progression?" Endocrine Reviews, vol. 33, no. 6, pp. 849-891, 2012.

[27] K. Dezaki, H. Hosoda, M. Kakei et al., "Endogenous ghrelin in pancreatic islets restricts insulin release by attenuating $\mathrm{Ca} 2+$ signaling in $\beta$-cells: Implication in the glycemic control in rodents," Diabetes, vol. 53, no. 12, pp. 3142-3151, 2004.

[28] K. Dezaki, "Ghrelin function in insulin release and glucose metabolism," The Ghrelin System, vol. 25, pp. 135-143, 2013.
[29] J. Tong, R. L. Prigeon, H. W. Davis, M. Bidlingmaier, M. H. Tschöp, and D. D’Alessio, "Physiologic concentrations of exogenously infused ghrelin reduces insulin secretion without affecting insulin sensitivity in healthy humans," The Journal of Clinical Endocrinology \& Metabolism, vol. 98, no. 6, pp. 2536-2543, 2013.

[30] Derek Lam, Devon Harris, and Zhenyu Qin, "Inflammatory Mediator Profiling Reveals Immune Properties of Chemotactic Gradients and Macrophage Mediator Production Inhibition during Thioglycollate Elicited Peritoneal Inflammation," Mediators of Inflammation, vol. 2013, Article ID 931562, 9 pages, 2013.

[31] E. E. McGrath, H. M. Marriott, A. Lawrie et al., “TNF-related apoptosis-inducing ligand (TRAIL) regulates inflammatory neutrophil apoptosis and enhances resolution of inflammation," Journal of Leukocyte Biology, vol. 90, no. 5, pp. 855-865, 2011.

[32] L. Lin, J. H. Lee, E. D. Buras et al., "Ghrelin receptor regulates adipose tissue inflammation in aging," $A G I N G$, vol. 8, no. 1, pp. 178-191, 2016.

[33] D. Baatar, K. Patel, and D. D. Taub, "The effects of ghrelin on inflammation and the immune system," Molecular and Cellular Endocrinology, vol. 340, no. 1, pp. 44-58, 2011.

[34] C. Cheyuo, R. Wu, M. Zhou, A. Jacob, G. Coppa, and P. Wang, "Ghrelin suppresses inflammation and neuronal nitric oxide synthase in focal cerebral ischemia via the vagus nerve," Shock, vol. 35, no. 3, pp. 258-265, 2011.

[35] X. Ma, L. Lin, J. Yue et al., "Ghrelin receptor regulates HFCSinduced adipose inflammation and insulin resistance," Nutrition \& Diabetes, vol. 3, no. 12, p. e99, 2013.

[36] Y. Pang, Y. Li, Y. Lv et al., "Intermedin restores hyperhomocysteinemia-induced macrophage polarization and improves insulin resistance in mice," The Journal of Biological Chemistry, vol. 291, no. 23, pp. 12336-12345, 2016.

[37] Z. Li, G. Xu, Y. Qin et al., "Ghrelin promotes hepatic lipogenesis by activation of mTOR-PPAR signaling pathway," Proceedings of the National Acadamy of Sciences of the United States of America, vol. 111, no. 36, pp. 13163-13168, 2014.

[38] J. M. Zigman, Y. Nakano, R. Coppari et al., "Mice lacking ghrelin receptors resist the development of diet-induced obesity," The Journal of Clinical Investigation, vol. 115, no. 12, pp. 3564-3572, 2005.

[39] Y. Sun, N. F. Butte, J. M. Garcia, and R. G. Smith, “Characterization of adult ghrelin and ghrelin receptor knockout mice under positive and negative energy balance," Endocrinology, vol. 149, no. 2, pp. 843-850, 2008. 


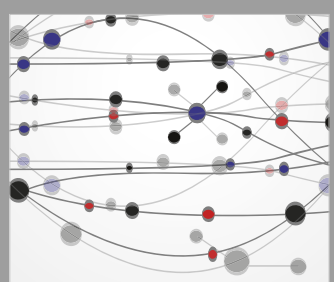

The Scientific World Journal
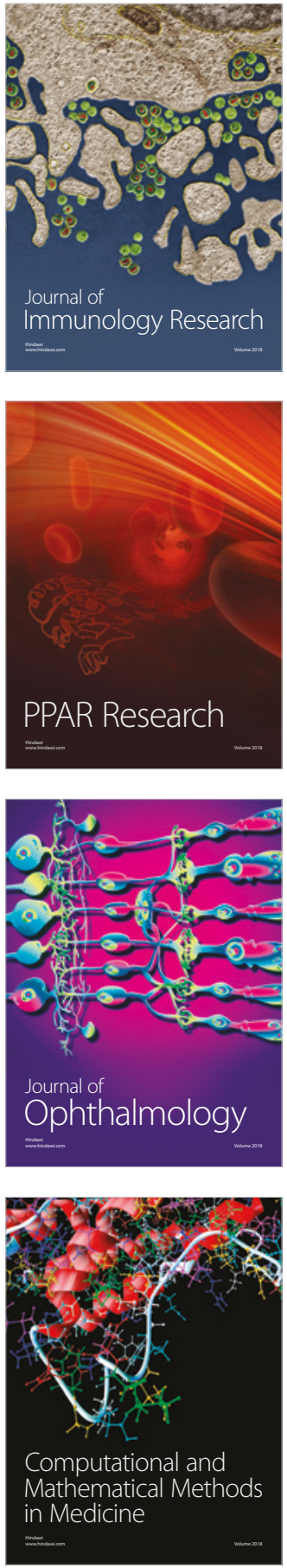

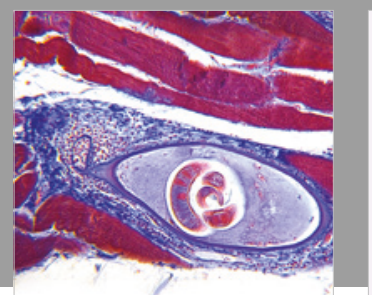

Gastroenterology Research and Practice

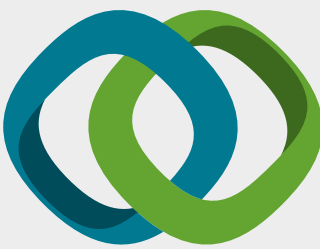

\section{Hindawi}

Submit your manuscripts at

www.hindawi.com
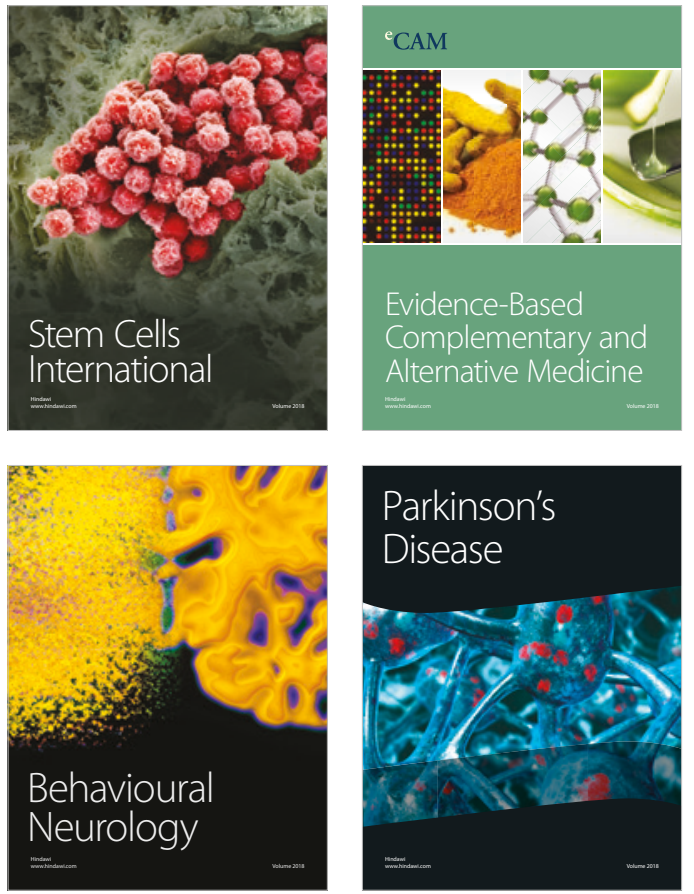

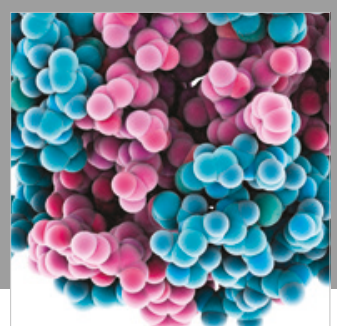

ournal of

Diabetes Research

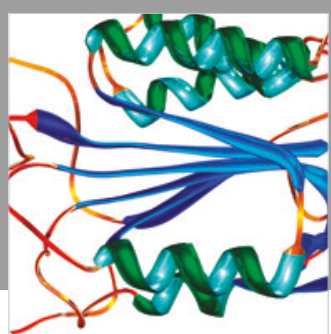

Disease Markers
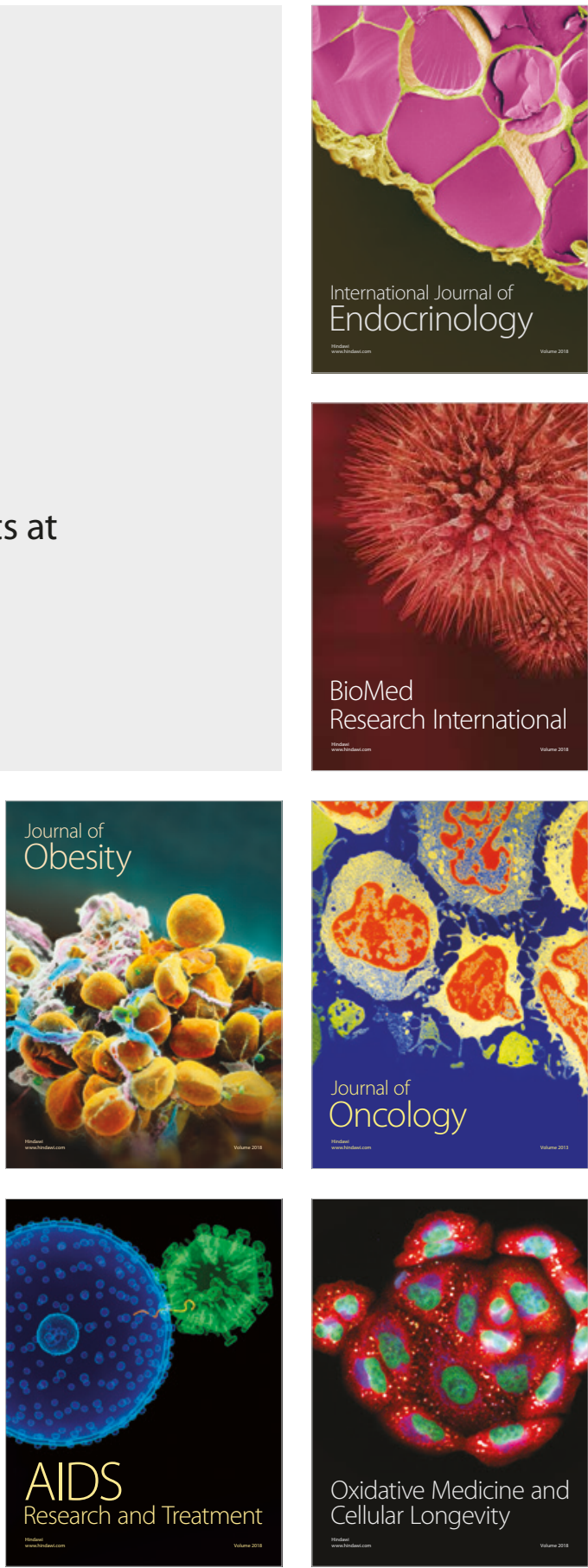